\title{
Middle Cranial Fossa (MCF) Approach without the use of Lumbar Drain for the Management of Spontaneous Cerebral Spinal Fluid (CSF) Leaks
}

Running head: MCF CSF Leaks

Rick F. Nelson, MD, PhD

Joseph P. Roche, MD

Bruce J. Gantz, MD

Marlan R. Hansen, MD

Author Affiliations: Department of Otolaryngology — Head and Neck Surgery (Dr. Nelson), Indiana University School of Medicine, Indianapolis, U.S.A. Departments of Otolaryngology-Head and Neck Surgery (Drs Roche, Gantz, Hansen) and Neurosurgery (Drs. Gantz \& Hansen), University of Iowa Hospitals and Clinics, Iowa City U.S.A.

\section{Corresponding Author:}

Rick F. Nelson, MD, PhD, Department of Otolaryngology - Head and Neck Surgery, Indiana University Health, 355 W. $16^{\text {th }}$ St. Suite 3200 , Indianapolis, IN 46202

Telephone: 317-963-7073

Fax: 317-963-7085

ricnelso@iupui.edu

Financial disclosures: none

Authors disclose no conflict of interest

This is the author's manuscript of the article published in final edited form as:

Nelson, R. F., Roche, J. P., Gantz, B. J., \& Hansen, M. R. (2016). Middle Cranial Fossa (MCF) Approach Without the Use of Lumbar Drain for the Management of Spontaneous Cerebral Spinal Fluid (CSF) Leaks. Otology \& Neurotology, 37(10), 1625-1629. https://doi.org/10.1097/MAO.0000000000001208 
1 Objective: To determine the efficacy and morbidity of repairing spontaneous cerebrospinal

2 fluid (CSF) leaks with the middle cranial fossa (MCF) approach without the use of a lumbar

3 drain $(\mathrm{LD})$, as perioperative use of $\mathrm{LD}$ remains controversial.

4

5 Study Design: Retrospective review from 2003-2015

6

Setting: University of Iowa Hospitals and Clinics and Indiana University Health Center

8

Patients: Those with a confirmed lateral skull base spontaneous CSF leaks and/or encephaloceles.

Intervention: MCF approach for repair of spontaneous CSF leak and/or encephalocele without the use of lumbar drain. Assessment of patient age, sex, body mass index (BMI), and medical comorbidities

Main Outcome Measure: Spontaneous CSF leak patient characteristics (age, sex, BMI, obstructive sleep apnea) were collected. Length of stay (LOS), hospital costs, post-operative complications, CSF leak rate, and need for LD were calculated.

Results: 65 operative MCF repairs were performed for spontaneous CSF leaks on 60 patients (5 had bilateral CSF leaks). CSF diversion with LD was used in 15 of 60 patients, mostly prior to 2010. After 2010, only 3 of 44 patients (6.7\%) had post-operative otorrhea requiring LD. The use of LD resulted in significantly longer LOS (3.6 \pm 1.6 vs. $8.7 \pm 2.9$ days) and hospital costs $(\$ 29,621)$. There were no postoperative complications in 77\% (50 of 65) of cases. Three cases required return to the operating room for complications including frontal subdural hematoma (1), subdural CSF collection (1) and tension pneumocephalus (1). No patients experienced long-term neurologic sequelae or long-term CSF leak recurrence with an average length of follow up of 19.5 months (range 3-137 months). The average patient BMI was $37.5 \pm 8.6 \mathrm{~kg} / \mathrm{m}^{2}$. The average age was $57.5 \pm 11.4$ years and $68 \%$ were female. Obstructive sleep apnea was present in $43.3 \%$ (26 of 60 ) of patients. 
31 Conclusions: The morbidity of the MCF craniotomy for repair of spontaneous CSF leaks is low 32 and the long-term efficacy of repair is high. Universal use of peri-operative lumbar drain is not 33 indicated and significantly increases length of stay and hospital costs. Obesity and obstructive 34 sleep apnea are highly associated with spontaneous CSF leaks.

35

36 
Spontaneous cerebrospinal fluid (CSF) leaks of the lateral skull base occur through defects of the dura and the lateral skull base (also known as the tegmen) overlying the pneumatized middle ear and mastoid. In addition, brain tissue can herniate through the bony defect resulting in an encephalocele. CSF leaks are termed spontaneous when there is no preceding trauma, surgery, or inciting cause.

The pathologic mechanism of spontaneous CSF leaks is still not well understood. There is a strong association between obesity and spontaneous CSF leaks (1-3). As the obesity epidemic increased between 1980 and 2010, the number of spontaneous CSF leak repairs has nearly doubled in the past decade (4). Though not seen in all obese patients, those with spontaneous CSF leaks have thinning of the skull base in addition to the skull calverium (3). This finding implicates an additional 'obesity-associated' intracranial process leading to skull thinning (3). This process could be due to elevated intracranial pressure. Chronically elevated intracranial pressure (ICP) over many years may lead to erosion of the skull, including the skull base. Spontaneous CSF leaks are associated with idiopathic intracranial hypertension (IIH), also known as benign intracranial hypertension or pseudotumor cerebri (5). Interestingly, measurements of CSF pressures during lumbar puncture in patients undergoing repair of spontaneous CSF leak show that only $10-36 \%$ of patients have elevated ICP $(6,7)$. Alternatively, it is possible that there are transient spikes in ICP that may not be detected on routine lumbar puncture. It is known that obstructive sleep apnea (OSA) is highly associated with obesity $(8,9)$ and OSA patients have transient large spikes in ICP during apnea spells $(10,11)$. We have Spontaneous CSF leaks have a very low rate of spontaneous closure. Surgical approaches to repair temporal bone spontaneous CSF leaks include middle cranial fossa (MCF) craniotomy (12-17), transmastoid (TM) $(2,7,18-20)$ or a combined (MCF/TM) $(1,21,22)$ approach. The 66 transmastoid approach is appealing as it avoids the risks of a craniotomy. However, the 67 recurrence rate of CSF leaks after transmastoid approach ranges from 6-20\% (7,20). In addition, 
68 the MCF approach allows for a more comprehensive evaluation of the entire tegmen, since up to $6950 \%$ of patients have multiple tegmen defects (15). The reported perioperative risks of MCF

70 approach are low, but can include hematomas, stroke and seizures.

71

72 In addition, the use of CSF diversion through the use of a lumbar drain (LD) during the repair of 73 spontaneous CSF leaks is controversial. The use of LDs during endoscopic repair of anterior 74 skull base CSF leaks has been studied and found to not reduce the rates of recurrent CSF leaks 75 (23,24). There is currently no consensus on if and when to use a LD for spontaneous CSF leak 76 repairs from the temporal bone. In addition, LD utilization has been associated with increased 77 complication rate of up to $12.3 \%$, including pneumocephalus, persistent headaches, meningitis, 78 uncal herniation and lumbar radiculopathy (25).

80 Here we report a large cohort of patients who have undergone MCF repair for spontaneous CSF 81 leaks. We review our perioperative complications of the MCF approach to repair spontaneous 82 CSF leaks. Also, we determine the effectiveness of the MCF repair without the use of lumbar 83 drains and the long-term stability of the repair.

84

85

86

87 
MATERIALS AND METHODS

89 Retrospective evaluation and data collection of patients were approved by the institutional review boards at the University of Iowa (\#201307714) and Indiana University Health (\#49109189). Patients were treated by 3 neurotologists (BJG, MRH, RFN) with spontaneous

92 CSF leaks from 2003 to 2015. CSF leaks were considered spontaneous when there was persistent clear otorrhea associated with tegmen defect(s) noted on high resolution CT IAC imaging and no previous history of skull base surgery, trauma or skull base fracture.

The following patient characteristics were documented from the medical record: (1) body mass index (BMI) calculated as kilograms per square meter $\left(\mathrm{kg} / \mathrm{m}^{2}\right)$, (2) age, (3) sex, (4) obstructive sleep apnea. Long term follow up was calculated from the date of operation to the last recorded clinic visit in the medical record. Complications were recorded by review of clinic notes in the medical record. For adults aged 20 years and older, obesity was defined as a BMI of 30.0 or higher (26). Obstructive sleep apnea was confirmed if the patient had (in the chart or per patient report) a positive polysomnogram (apnea-hypopnea index > 5) or was using continuous positive airway pressure (CPAP) or bilevel positive airway pressure (BiPAP). Patients without the clinical diagnosis of OSA were not independently tested. However, starting in 2014, all patients at Indiana University Health with spontaneous CSF leaks were prospectively tested for OSA with polysomnogram. Differences in means were tested with a 2-tailed T test with significance set to $\alpha \leq 0.05$.

All patients were treated with an MCF approach. Briefly, a pterional incision was performed with harvesting of a large temporalis fascia graft. The temporalis muscle was incised to leave a cuff of muscle and fascia attached to the calvarium and the muscle was reflected anteriorly. A 4.5 x 4.5 craniotomy was performed over the middle ear and mastoid. Intraoperative mannitol (0.5 gram per kilogram) was administered and the patient was hyperventilated to end-tidal $\mathrm{CO}_{2}$ $<30$. The temporal lobe dura was elevated and the dura and skull base defects were identified. The entire floor of the middle fossa was explored including anterior petrous apex. Encephaloceles were amputated and removed with bipolar electrocautry when present. Reconstruction consisted of a multilayer closure with a fascia soft tissue seal on the skull base, split calvarial bone grafts over bone defects and collagen matrix abutting the native dural defects. 
119 Some cases were repaired with intradural placement of collagen matrix. The craniotomy was

120 replaced and the muscle and galeal layers were sutured in a water-tight fashion. The patient was

121 extubated after the case and ambulation commenced on post-operative day 1.

122

123 Lumber drains were placed if there was persistent otorrhea or CSF rhinorrhea on post-operative

124 days 2 or 3. Many patients had an ipsilateral pressure equalizing tube typically placed by an

125 outside otolaryngologist prior to the diagnosis. CSF was drained at $10 \mathrm{ml} /$ hour for 5 days when

126 LD was used. In our cohort of patients, we did not routinely use diuretics or acetazolamide to

127 manage CSF pressure in the post-operative period.

128

129 Procedural and hospital costs were estimated based upon previous charges for spontaneous CSF 130 leak patients with lumbar drains using 2016 data. These estimated charges can vary based upon 131 patient comorbidities and hospital location. These amounts are specified as billed charges and do 132 not reflect actual amount reimbursed or paid as these later amounts are variable depending upon 133 insurance coverage. The charges for lumbar drain placed at bedside (CPT 62272) are for the 134 device and facility charges and does not include surgeon fees. The daily hospital charges include 135 progressive care unit facility charges. 


\section{RESULTS}

We examined medical records of 60 patients (65 cases) from 2 academic medical centers who underwent MCF repair of spontaneous CSF leaks of the temporal bone from 2007 to 2015. Five (5) patients had bilateral temporal bone CSF leaks. Figure 1 is representative CT image of patient with a dehiscent tegmen mastoidium and fluid filling the mastoid (Figure 1A). Many patients have multiple areas of tegmen dehiscence (Figure 1B).

The average age was $57.5 \pm 11.4$ years and $68.3 \%$ of the patients were female (Table 1). Most patients $(83.3 \%)$ were overweight $\left(\mathrm{BMI}>25 \mathrm{~kg} / \mathrm{m}^{2}\right)$ and the average BMI was $37.5 \pm 8.7 \mathrm{~kg} / \mathrm{m}^{2}$ (Table 1). The rate of obstructive sleep apnea was $43.3 \%$ (Table 1). Many patients were not prospectively tested for OSA. Starting in 2014, all patients at Indiana University Health with spontaneous CSF leaks underwent polysomnogram and all patients (6 of 6) had OSA.

There were no perioperateive complications in 77\% (50 of 65) of the cases. The most common complication was persistent otorrhea noted on post-operative days 2 or 3 (Table 2). In all such cases, a LD drain was placed for 5 days and the otorrhea resolved. There were 2 cases of mental status change with temporal lobe edema that resolved with steroid treatment. Three cases required return to the operating room. One patient developed a subdural hematoma that required a burr hole evacuation. Another patient developed a frontal subdural CSF fluid collection after hospital discharge that required burr hole evacuation. Lastly, one patient developed tension pneumocephalus that required return to the operating room for evacuation. Intraoperatively it was noted that the split calvarial bone grafts had not formed a tight seal on the middle fossa floor resulting in a 'ball valve' effect leading to trapping air intracranially. The temporalis fascia was placed over the skull base to form a soft tissue seal, prior to replacement of the bone grafts. All 3 patients recovered fully. Isolated cases of delayed facial paralysis, atrial fibrillation, meningitis and seizure were treated medically and patients demonstrated full recovery. There were no cases of ipsilateral CSF leak at last follow up (Table 2).

\section{Early in the study, prior to 2010, 21 patients underwent MCF CSF leak repair. Approximately} half of the patients had lumber drains placed intra-operatively during MCF repair and the other half did not have a lumber drain. There were no post-operative CSF leaks in either group 
168 suggesting that lumber drain placement may not be necessary for a successful repair. Thus,

169 starting in 2010, we performed 44 MCF repairs of spontaneous CSF leaks with the intention of 170 not using a perioperative LD. After 2010, only 3 of 44 patients (6.7\%) has post-operative 171 otorrhea requiring LD. The average length of stay for patients without LD was significantly 172 shorter than those who had a LD placed preoperatively (3.6 \pm 1.6 vs. $8.7 \pm 2.9$ days; $\mathrm{P}<0.01$; 173 Table 3). In addition, the use of lumbar drains increases cost. We estimated the cost (in 2016 174 dollars) to be $\$ 13,316$ for placement of the LD and $\$ 3,261$ per day for hospital room and nursing 175 care. Thus, for 5 additional hospital days and LD, the added cost is approximately $\$ 29,621$

176 (Table 3).

177

178 


\section{DISCUSSION}

Here we show that the MCF approach to repair temporal bone spontaneous CSF leaks is highly effective and is safe. The use of a LD is not required for successful repair in most cases. Preemptive use of LDs increases length of stay and cost.

Consistent with other previous reports $(1,2,4)$, we find a strong association between spontaneous CSF leaks and obesity. The obesity epidemic developed in the United States starting in the 1990s (27). The percentage of American that are obese (BMI $\geq 30)$ has risen from $12 \%$ in 1991 to $19.8 \%$ in 2000 to $29 \%$ in 2010 (28). The mechanism of how obesity could lead to skull thinning remains unknown. Previously, we showed that patients with spontaneous CSF leaks were obese and had thinning of the calvarium in addition to the skull base, while a control group of obese patients without spontaneous CSF leaks did not have thinning of the calvarium. Because obesity is highly associated with spontaneous CSF leaks, these data suggest that there is another obesityassociated factor which contributes to skull thinning and spontaneous CSF leaks. This obesityassociated factor likely leads to elevated intracranial pressure either chronically or on an intermittent basis.

Spontaneous CSF leaks are associated with IIH (5), and some patients ( $36 \%)$ have elevated ICP during lumbar puncture (6). This shows that not all patients with spontaneous CSF leaks have elevated baseline ICP, and other factors may contribute to transient elevations in ICP. Patients with spontaneous CSF leaks have a high rate of OSA and patients with OSA have been found to have elevations in ICP and arterial blood pressure during apnea events (10). It is postulated that apneas lead to hypercarbia, which in turn leads to cerebral vasodilation and elevated ICP. Our data show that patients with spontaneous CSF leaks have a much higher prevalence of OSA $(\sim 43 \%)$ than the national average. In addition, these patients presented with the diagnosis of OSA and many had not had a polysomnogram. Thus, this rate may underestimate the true rate of OSA in patients with spontaneous CSF leaks. Since 2014, we have tested all patients with spontaneous CSF leaks for sleep apnea with a polysomnogram.

Superior semicircular canal dehiscence (SSCD) has been associated with tegmen erosion $(29,30)$ and the surgeon should be alerted to the possibility of SSCD as the dura is elevated from the 
middle fossa floor. In this study, we did not specifically evaluate or repair SSCD unless the patient had vestibular symptoms consistent with SSCD (i.e. Tullio's phenomena). However, during the repair we resurface the SSC as we resurface the skull base with soft tissue and bone grafts.

Several previous reports have used LDs during MCF repair of temporal bone spontaneous CSF leaks $(15,17,21,22)$ with postoperative CSF leaks rates of 4-9\%. Since 2010 we have not used LD during our MCF CSF leak repairs and our post-operative leak rate in 44 patients was $6.7 \%$. In addition, we estimated that the added cost of a LD and 5 days in the hospital to be close to $\$ 60,000$. Given that it is not necessary in the vast majority of cases, we advocate against the preemptive use of LDs for spontaneous CSF leaks. A review study using LD for anterior skull base CSF leaks also failed to show significant benefit in CSF leak repair (24).

The vast majority of patients who underwent MCF CSF leak repair had no postoperative complications. The most common complication was a postoperative CSF leak that was managed effectively with a LD. We placed the LD relatively early in the postoperative period (day 2-3) if there was persistent otorrhea. While it is possible that some of these cases may have gone on to resolve without the need for a $\mathrm{LD}$ if they had been observed longer, we favored early intervention in these cases. There were no long term sequela from using the MCF approach.

There are inherent limitations to this study. By design the study is retrospective and nonrandomized. In addition, increased surgeon experience over time could account for a decreased post-operative CSF leak rate. Finally, the generalizability of the results to all centers irrespective of surgical experience with MCF approach may not be possible.

Thus, we find the morbidity of the MCF craniotomy for repair of spontaneous CSF leaks is low and the long-term efficacy of repair is high. The universal preoperative placement of LD does not appear to be indicated. We advocate for LD only in the infrequent case of post-operative otorrhea. Use of LD significantly increases length of stay and hospital costs. Finally, we also advocate for all patients with spontaneous CSF leaks to be tested for OSA. 


\section{FIGURE LEDGENDS}

242

243 Figure 1: A. Representative coronal temporal bone CT scan image demonstrating a right tegmen 244 mastoideum defect with fluid in the mastoid. B. Intraoperative view of a left middle fossa floor 245 showing multiple tegmen defects (arrows). $\mathrm{AE}=$ arcuate eminence, $\mathrm{L}=$ Lateral, $\mathrm{M}=\mathrm{Medial}, \mathrm{A}$ $246=$ Anterior, $\mathrm{P}=$ Posterior.

247

248 Table 1: Patient characteristic; $B M I=$ Body Mass Index; $\mathrm{kg}=$ kilograms; $\mathrm{m}=$ meter

249

250

Table 2: Post-operative Complications (65 cases). * = required return to the operating room 251

252

Table 3: Lumbar Drain Use and Estimated Cost

253

254

255

256

257 


\section{REFERENCES}

1. Stucken EZ, Selesnick SH, Brown KD. The role of obesity in spontaneous temporal bone encephaloceles and CSF leak. Otology \& neurotology : official publication of the American Otological Society, American Neurotology Society [and] European Academy of Otology and Neurotology 2012;33:1412-7.

2. LeVay AJ, Kveton JF. Relationship between obesity, obstructive sleep apnea, and spontaneous cerebrospinal fluid otorrhea. The Laryngoscope 2008;118:275-8.

3. Nelson RF, Hansen KR, Gantz BJet al. Calvarium Thinning in Patients with Spontaneous Cerebrospinal Fluid Leak. Otology \& neurotology : official publication of the American Otological Society, American Neurotology Society [and] European Academy of Otology and Neurotology 2014.

4. Nelson RF, Gantz BJ, Hansen MR. The Rising Incidence of Spontaneous Cerebrospinal Fluid Leaks in the United States and the Association With Obesity and Obstructive Sleep Apnea. Otology \& neurotology : official publication of the American Otological Society, American Neurotology Society [and] European Academy of Otology and Neurotology 2014.

5. Schlosser RJ, Woodworth BA, Wilensky EMet al. Spontaneous cerebrospinal fluid leaks: a variant of benign intracranial hypertension. The Annals of otology, rhinology, and laryngology 2006;115:495-500.

6. Allen KP, Perez CL, Kutz JWet al. Elevated intracranial pressure in patients with spontaneous cerebrospinal fluid otorrhea. The Laryngoscope 2013.

7. Kim L, Wisely CE, Dodson EE. Transmastoid Approach to Spontaneous Temporal Bone Cerebrospinal Fluid Leaks: Hearing Improvement and Success of Repair. Otolaryngology--head and neck surgery : official journal of American Academy of Otolaryngology-Head and Neck Surgery 2014.

8. Li C, Ford ES, Zhao Get al. Prevalence of self-reported clinically diagnosed sleep apnea according to obesity status in men and women: National Health and Nutrition Examination Survey, 20052006. Preventive medicine 2010;51:18-23.

9. Lee W, Nagubadi S, Kryger MHet al. Epidemiology of Obstructive Sleep Apnea: a Populationbased Perspective. Expert review of respiratory medicine 2008;2:349-64.

10. Jennum P, Borgesen SE. Intracranial pressure and obstructive sleep apnea. Chest 1989;95:27983.

11. Sugita Y, lijima S, Teshima Yet al. Marked episodic elevation of cerebrospinal fluid pressure during nocturnal sleep in patients with sleep apnea hypersomnia syndrome.

Electroencephalography and clinical neurophysiology 1985;60:214-9.

12. Gacek RR, Gacek MR, Tart R. Adult spontaneous cerebrospinal fluid otorrhea: diagnosis and management. The American journal of otology 1999;20:770-6.

13. Brown NE, Grundfast KM, Jabre Aet al. Diagnosis and management of spontaneous cerebrospinal fluid-middle ear effusion and otorrhea. The Laryngoscope 2004;114:800-5.

14. Leonetti JP, Marzo S, Anderson Det al. Spontaneous transtemporal CSF leakage: a study of 51 cases. Ear, nose, \& throat journal 2005;84:700, 2-4, 6.

15. Gubbels SP, Selden NR, Delashaw JB, Jr.et al. Spontaneous middle fossa encephalocele and cerebrospinal fluid leakage: diagnosis and management. Otology \& neurotology : official publication of the American Otological Society, American Neurotology Society [and] European Academy of Otology and Neurotology 2007;28:1131-9.

16. Kutz JW, Jr., Husain IA, Isaacson Bet al. Management of spontaneous cerebrospinal fluid otorrhea. The Laryngoscope 2008;118:2195-9. 
17. Vivas EX, McCall A, Raz Yet al. ICP, BMI, Surgical Repair, and CSF Diversion in Patients Presenting With Spontaneous CSF Otorrhea. Otology \& neurotology : official publication of the American Otological Society, American Neurotology Society [and] European Academy of Otology and Neurotology 2014;35:344-7.

18. Kari E, Mattox DE. Transtemporal management of temporal bone encephaloceles and CSF leaks: review of 56 consecutive patients. Acta oto-laryngologica 2011;131:391-4.

19. Oliaei S, Mahboubi H, Djalilian HR. Transmastoid approach to temporal bone cerebrospinal fluid leaks. American journal of otolaryngology 2012;33:556-61.

20. Stevens SM, Rizk HG, Mcllwain WRet al. Association between Lateral Skull Base Thickness and Surgical Outcomes in Spontaneous CSF Otorrhea. Otolaryngology--head and neck surgery : official journal of American Academy of Otolaryngology-Head and Neck Surgery 2016;154:70714.

21. Kenning TJ, Willcox TO, Artz GJet al. Surgical management of temporal meningoencephaloceles, cerebrospinal fluid leaks, and intracranial hypertension: treatment paradigm and outcomes. Neurosurgical focus 2012;32:E6.

22. Son HJ, Karkas A, Buchanan Pet al. Spontaneous Cerebrospinal fluid effusion of the temporal bone: Repair, audiological outcomes, and obesity. The Laryngoscope 2013.

23. Albu S, Emanuelli E, Trombitas Vet al. Effectiveness of lumbar drains on recurrence rates in endoscopic surgery of cerebrospinal fluid leaks. American journal of rhinology \& allergy 2013;27:e190-4.

24. Bakhsheshian J, Hwang MS, Friedman M. What is the evidence for postoperative lumbar drains in endoscopic repair of CSF leaks? The Laryngoscope 2015;125:2245-6.

25. Stokken J, Recinos PF, Woodard Tet al. The utility of lumbar drains in modern endoscopic skull base surgery. Current opinion in otolaryngology \& head and neck surgery 2015;23:78-82.

26. Clinical guidelines on the identification, evaluation, and treatment of overweight and obesity in adults: executive summary. Expert Panel on the Identification, Evaluation, and Treatment of Overweight in Adults. The American journal of clinical nutrition 1998;68:899-917.

27. Mokdad AH, Bowman BA, Ford ESet al. The continuing epidemics of obesity and diabetes in the United States. JAMA : the journal of the American Medical Association 2001;286:1195-200.

28. Mokdad AH, Serdula MK, Dietz WHet al. The spread of the obesity epidemic in the United States, 1991-1998. JAMA : the journal of the American Medical Association 1999;282:1519-22.

29. Nadaraja GS, Gurgel RK, Fischbein NJet al. Radiographic evaluation of the tegmen in patients with superior semicircular canal dehiscence. Otology \& neurotology : official publication of the American Otological Society, American Neurotology Society [and] European Academy of Otology and Neurotology 2012;33:1245-50.

30. Rizk HG, Hatch JL, Stevens SMet al. Lateral Skull Base Attenuation in Superior Semicircular Canal Dehiscence and Spontaneous Cerebrospinal Fluid Otorrhea. Otolaryngology--head and neck surgery : official journal of American Academy of Otolaryngology-Head and Neck Surgery 2016. 

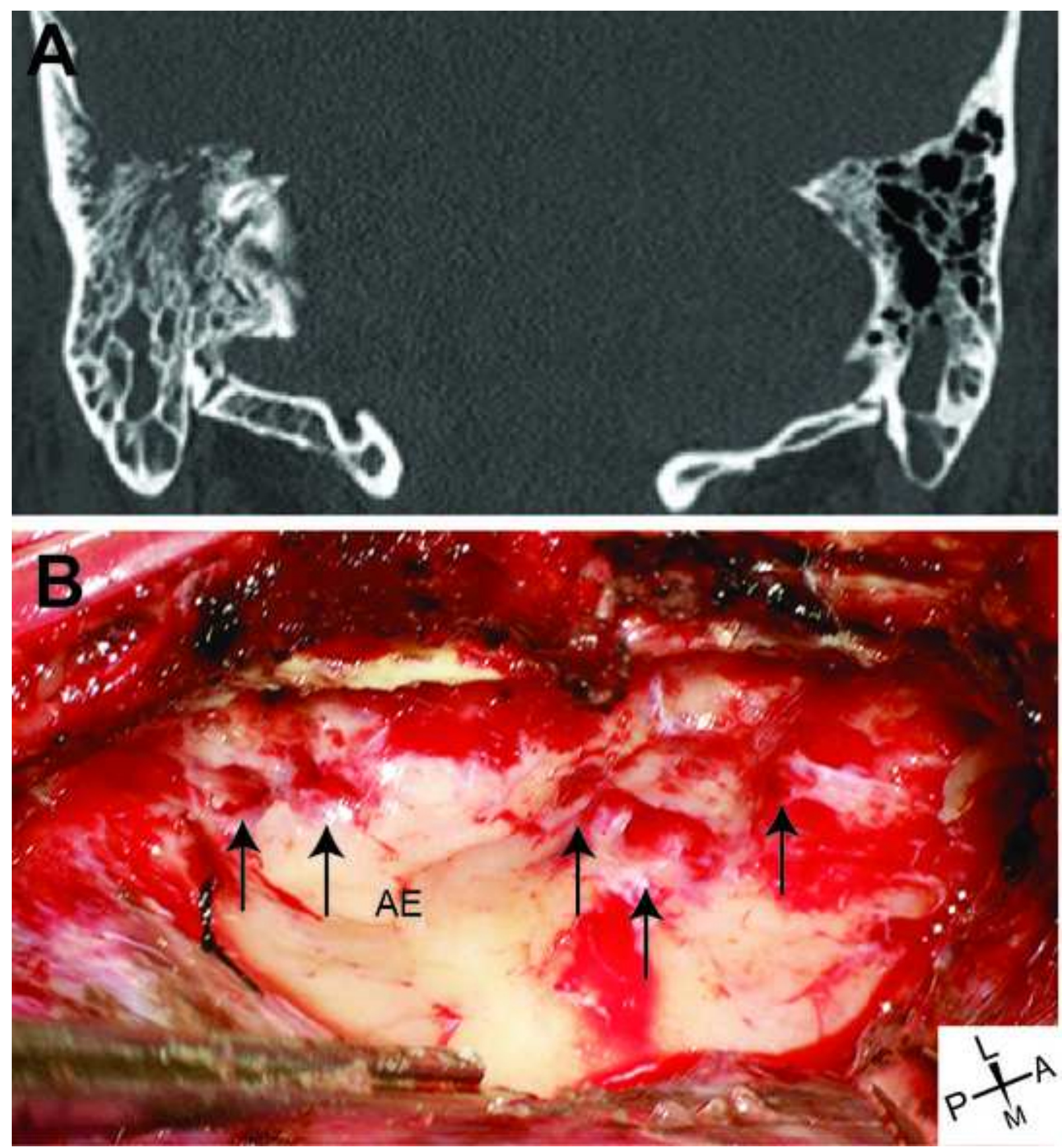


\begin{tabular}{|c|c|}
\hline Gender & Number (\%) \\
\hline Male & 19 (31.7\%) \\
\hline \multirow[t]{2}{*}{ Female } & $41(68.3 \%)$ \\
\hline & Total $=60$ \\
\hline Age (Years) & Number (\%) \\
\hline $21-30$ & $1(1.7 \%)$ \\
\hline $31-40$ & $6(10.0 \%)$ \\
\hline $41-50$ & $6(10.0 \%)$ \\
\hline $51-60$ & $18(30.0 \%)$ \\
\hline $61-70$ & $23(38.3 \%)$ \\
\hline $71-80$ & 5 (8.3\%) \\
\hline $81-90$ & $1(1.7 \%)$ \\
\hline \multicolumn{2}{|l|}{ Average $=57.5 \pm 11.4$} \\
\hline BMI $\left(\mathrm{kg} / \mathrm{m}^{2}\right)$ & Number (\%) \\
\hline$\leq 24.99$ & $2(3.3 \%)$ \\
\hline $25.00-29.99$ & $8(13.3 \%)$ \\
\hline $30.00-34.99$ & $15(25.0 \%)$ \\
\hline $35.00-39.99$ & $13(21.7 \%)$ \\
\hline $40.00-44.99$ & $13(21.7 \%)$ \\
\hline $45.00-49.99$ & $5(8.3 \%)$ \\
\hline $50.00-54.99$ & $2(3.3 \%)$ \\
\hline $55.00-59.99$ & $0(0 \%)$ \\
\hline $60.00-64.99$ & $2(3.3 \%)$ \\
\hline $65.00-69.99$ & $1(1.7 \%)$ \\
\hline \multicolumn{2}{|l|}{ Average $=37.5 \pm 8.7$} \\
\hline Comorbidities & Number (\%) \\
\hline Obstructive Sleep Apnea & $26(43.3 \%)$ \\
\hline
\end{tabular}




\begin{tabular}{|l|l|}
\hline \multicolumn{1}{|c|}{ Complication (<30 days post-op) } & \multicolumn{2}{|c|}{ Number (\%) } \\
\hline Persistent otorrhea requiring LD & $5(7.7 \%)$ \\
\hline Confusion with Temporal Lobe Edema & $2(3.1 \%)$ \\
\hline Frontal Subdural Hematoma* & $1(1.5 \%)$ \\
\hline Subdural CSF Collection* & $1(1.5 \%)$ \\
\hline Tension Pneumocephalus* & $1(1.5 \%)$ \\
\hline Delayed Facial Paralysis & $1(1.5 \%)$ \\
\hline Meningitis & $1(1.5 \%)$ \\
\hline Seizure & $1(1.5 \%)$ \\
\hline Atrial Fibrillation & $1(1.5 \%)$ \\
\hline & \\
\hline Complication (>30 days post-op) & \\
\hline Wound drainage & $1(1.5 \%)$ \\
\hline Complex partial seizure & $1(1.5 \%)$ \\
\hline Word finding difficulty & $1(1.5 \%)$ \\
\hline Ipsilateral CSF leak & $0(0 \%)$ \\
\hline
\end{tabular}




\begin{tabular}{|c|c|}
\hline $\begin{array}{c}\text { Perioperative Lumbar Drain } \\
\text { (\# cases) }\end{array}$ & Length of stay (days) \\
\hline No (50) & $3.6+/-1.6$ \\
\hline Yes (15) & $8.7+/-2.9$ \\
\hline & \\
\hline Type of Charge & Cost (estimated) \\
\hline Lumbar drain & $\$ 13,316$ \\
\hline Hospital Room + Nursing & $\$ 3,261$ (per day) \\
\hline $\mathbf{5}$ day total charge & $\mathbf{\$ 2 9 , 6 2 1}$ \\
\hline
\end{tabular}

